\title{
MULTILAYER ELECTRODEPOSITION FROM MICROMACHINED TEMPLATES: A CONTROLLABLE, SCALABLE ROUTE TO HIERARCHICAL SUPERHYDROPHOBIC SURFACES
}

\author{
M. Kim ${ }^{1 *}$, J. Kim², and M. G. Allen ${ }^{1}$ \\ ${ }^{1}$ University of Pennsylvania, Philadelphia, Pennsylvania, USA \\ ${ }^{2}$ Georgia Institute of Technology, Atlanta, Georgia, USA
}

\begin{abstract}
We present multilayer electrodeposition guided by micromachined templates as a route to controllable, scalable realization of superhydrophobic surfaces. Sequential electrodeposition of nickel/copper multilayers, followed by a timed, selective copper etching creates metallic, dual-scale hierarchical surface structures, in which microscale pillar structures are defined by lithographically-patterned templates, and nanostructures (50-500 $\mathrm{nm})$ are defined by deposition process parameters. Two types of superhydrophobic surfaces are realized: (1) Metallic surfaces with silane-modified surfaces; (2) and elastomeric surfaces based on polydimethylsiloxane (PDMS) replica molding. Both surfaces exhibit strong non-wetting properties characterized by high static water contact angle reaching over $160^{\circ}$ and low contact angle hysteresis $\left(<10^{\circ}\right)$ due to their structural hierarchy. Self-cleaning properties of the fabricated surfaces are demonstrated.
\end{abstract}

\section{INTRODUCTION}

Superhydrophobic surfaces are essential for the survival of many biological systems, such as lotus leaf [1] and water strider [2]. Such non-wetting surfaces are usually characterized by an exceptionally high water contact angle, low contact angle hysteresis, and low sliding angle, i.e., the angle at which a droplet of a certain volume begins to "roll off" from the surface. Superhydrophobicity is useful for many MEMS applications, e.g., improved cooling based on enhanced nucleate boiling [3].

To fabricate superhydrophobic surfaces, applying materials with low intrinsic surface energy, as well as controlling their structural textures are important; even with the materials with extremely low surface energy (e.g., Teflon ${ }^{\mathrm{TM}}$ ), superhydrophobicity is not achieved if the material is perfectly smooth. Hydrophobic materials with multi-scale structural textures, comprising microscale $(1-100 \mu \mathrm{m})$ structures decorated with submicrometerscale protrusions, form minimized solid-to-water contact area, and thereby exhibit superhydrophobic properties that cannot be achieved from either smooth or textured surfaces with single-scale micro- or nanostructures [1].

Fabrication approaches for hierarchical, superhydrophobic surfaces are usually categorized into three types: bottom-up, topdown, and hybrid. Bottom-up approaches are typically based on colloidal synthesis [4] or sequential material growth/removal [5]. The major advantage of these approaches lies in their simplicity; however, it is relatively difficult to achieve designed wetting properties due to the spontaneous nature of the processes. On the contrary, both micro- and nanostructures can be precisely defined by top-down processes. Sequential lithography steps or pattern transfer processes (e.g., imprinting) are employed to create hierarchical surfaces with designed wettabilities such as surfaces with varying wettability in different orientations (i.e., anisotropic wettability [6]), or at different locations (i.e., surface wettability gradient [7]). However, realization of the nanostructures often requires the use of relatively intensive fabrication techniques, such as electron-beam lithography.
The present approach can be categorized as a hybrid approach, as it combines top-down microlithography and bottom-up multilayer electrodeposition to create structural hierarchy. Lithographically-defined templates are utilized to guide multilayer deposition, thereby creating metallic structures comprised of microscale pillars decorated with concentric ring-like nanostructures, the size scales of which are defined by the individual layer thicknesses of nickel and copper. Surface modification based on silane vaporization, or PDMS replica molding, leads to the realization of metallic or elastomeric hydrophobic surfaces, respectively.

Compared to other hybrid approaches [8,9], the multilayerbased approach has notable advantages. First, the orientation, location, and geometries of nanostructures are relatively well controlled compared to other bottom-up nanostructure synthesis approaches. Second, controlled nanostructures of high aspect ratios can be formed on the sidewalls of the microstructures, where even the state-of-the-art nanolithography-based approaches (e.g., focused ion-beam lithography, nanoimprinting) usually fail to form desired patterns. Sidewall features can be achieved through deep reactive ion etching of silicon; however, the aspect-ratios of such "scalloping" structures are limited. It is known that well-defined, sidewall nanostructures may substantially improve the robustness (or stability) of non-wetting state of structured surfaces [10]. Third, the present approach provides a route to metallic surfaces with controlled micro/nano features. The relatively robust nature of metal may lead to the use of these surfaces in harsh environments, such as underwater.

\section{FABRICATION APPROACH}

The present approach begins with the fabrication of a template comprising a $600 \mathrm{~nm}$-thick sputtered copper seed layer on a sacrificial-layer-coated silicon wafer, and a photoresist mold patterned on the seed (Figure 1(a)). A sequential dual-bath electrodeposition of nickel and copper follows (Figure 1(b)) to form multilayers. Individual layer thicknesses are dictated by control of deposition time, while the deposition currents in the baths are fixed. Typically, current densities of $12 \mathrm{~mA} / \mathrm{cm}^{2}$ are utilized in both nickel and copper deposition baths, resulting in deposition rates of $2.7 \mathrm{~nm} / \mathrm{s}$ and $3.3 \mathrm{~nm} / \mathrm{s}$, respectively. Further details regarding the deposition setup, multilayer growth modeling, and parameter studies (e.g., deposition characteristics of different types of deposition baths, spatial non-uniformity of the deposition) can be found in [11].

Initially, the multilayer growth is vertically confined within the photoresist mold. After the mold is filled, the growth progresses in three dimensions; the deposition is continued until the deposit is sufficiently thick to cover the photoresist. The layout is properly designed so that the current densities (and corresponding metal deposition rates) do not decrease more than $10 \%$ during this second stage of the deposition. The multilayer is mechanically delaminated from the silicon substrate (Figure 1(c)) using tweezers; the copper seed layer is easily detached from the sacrificial polymethylmethacrylate (PMMA) layer due to relatively weak polymer-to-metal adhesion. 


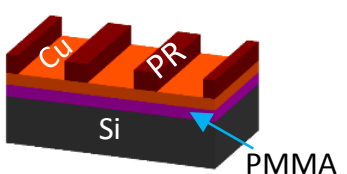

(a) Template fabrication

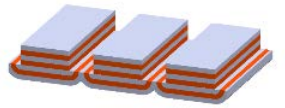

(c) Delamination \& template removal

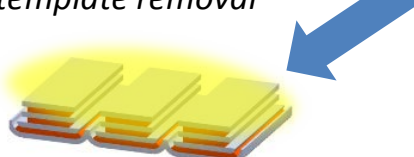

(e) Silane vapor dep.

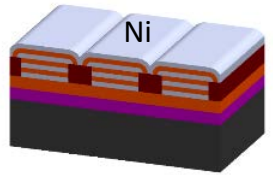

(b) Multilayer deposition

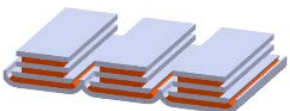

(d) Selective Cu etch

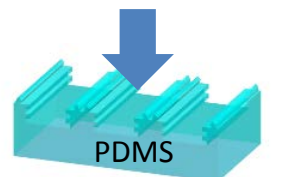

(f) PDMS replication
Figure 1. Fabrication procedure. Note the schematics are flipped upside down starting with step (c).

The seed and photoresist layers are sequentially removed in phosphorous-based etchant (Aluminum etchant Type A, Transene) and acetone, respectively. After the template is completely removed from the deposited multilayer, the multilayer "growth rings" are exposed where the concentric, surface relief nanostructures of designed nickel elevation (with respect to copper) are formed after a selective copper etch (Figure 1(d)). A thiourea-based etchant (1M of thiourea dissolved in water, $\mathrm{pH}$ tuned to 1 by hydrochloric acid) is used for the etching. The widths of protrusions and valleys of the nanostructures correspond to the thicknesses of nickel and copper, respectively. Large areas of surface relief structures through a single deposition process are achieved; fabricated samples were as large as $20 \mathrm{~cm}^{2}$.

It is important to note that both copper and nickel growths are isotropic throughout the deposition. As a result, the orientation of the resulting nanostructure protrusions after the copper etch is nearly normal to the surface (unless an extended period of etching is performed). This is confirmed by a cross-sectional image shown in Figure 2; the individual layers of the multilayer and the patterned photoresist were forming right angles prior to the delamination from
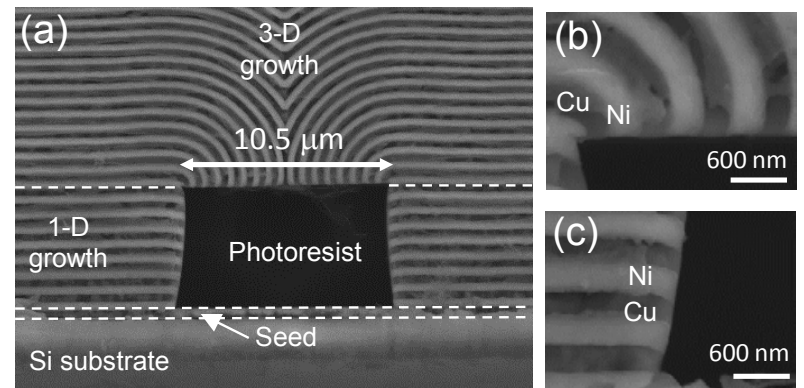

Figure 2. Cross-sectional view of a multilayer prior to the delamination. Growth of the layers is nearly normal to the surface of the photoresist mold. The periodicity of nickel and copper is approximately $600 \mathrm{~nm}$ for this sample. Figures (b) and (c) are magnified views of respective sites in (a). the substrate. Such well-defined surface relief structures enabled PDMS replica molding without de-molding issues (e.g., damaging either metal surface relief or cured PDMS nanostructures), leading to a single-step reproduction of hierarchical, non-wetting surfaces.

Two superhydrophobic surface types are fabricated from the relief structures that are originally hydrophilic. First, metallic superhydrophobic structures are formed by vacuum-assisted deposition of a silane monolayer on the multilayers (Figure 1(e)). Second, PDMS superhydrophobic structures are formed by replica molding using the silane-treated surface relief structures as master molds (Figure 1(f)). Experimentally, we found that PDMS with relatively high modulus should be employed to replicate both microand nanoscale features without de-molding issues. This is achieved by employing either (1) copolymer-based h-PDMS (tensile modulus $\sim 9 \mathrm{MPa}$ )[12], or (2) conventional Sylgard 184 (Dow Corning) with high crosslinker-to-prepolymer weight ratio (e.g., 1:4).

\section{DESIGNED AND FABRICATED SUPERHYDROPHOBIC SURFACES}

Hierarchical structures are properly designed and fabricated to realize superhydrophobic surfaces. Among various designs, a pillartype geometry is chosen for the microstructures, in order to minimize the areal fraction of solid-to-water contact. The designed heights of the microstructures $\left(h_{\text {micro }}\right)$ are larger than half their width ( $w$ micro) so that water has more of a chance to form a stable solidwater-air interface "on" the pillars [13]. Two types of structures were fabricated: microstructures alone (designated ' $M$ '), and microstructures decorated with nanostructures (designated ' $\mathrm{MN}$ '). The fabricated metallic hierarchical surfaces (Metal, MN) are comprised of micropillars with valleys textured on the nanoscale (Figure 3, Figure 4 (a)-(c)), while the PDMS structures (PDMS, $\mathrm{MN}$ ) have nanostructures present on the pillar protrusions (Figure 3, Figure 4(a),(d),(e)). Although relatively small periodicities of metallic nanostructures ( 200 nm) can be achieved (Figure 4(c)), the minimum achievable nanostructure periodicity of replicated PDMS surfaces is approximately 1 micrometer (with moderate aspect ratios ranging from 1:1 to 2:1) due to the difficulties of demolding. Corresponding single-scale microstructures (Metal, $\mathrm{M}$ and PDMS, M) are also fabricated for comparison.
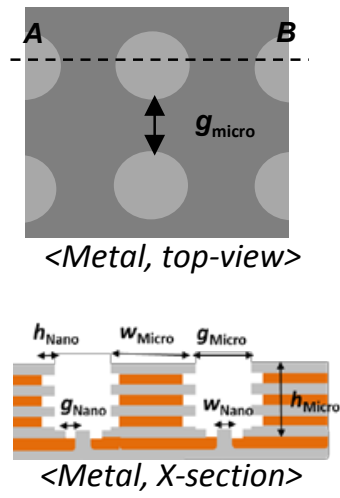
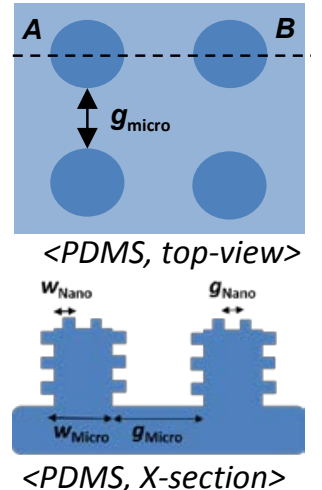

Figure 3. Schematic view of designed metal (left) and PDMS (right) hierarchical structures. 


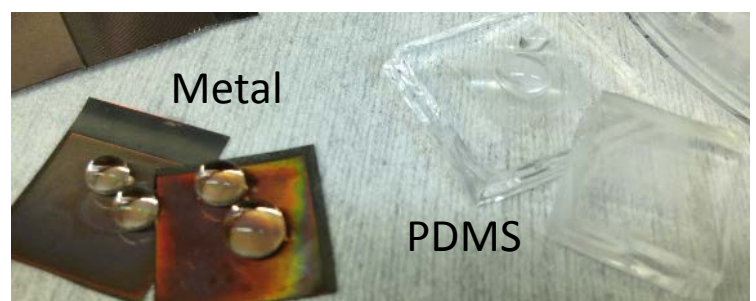

(a)

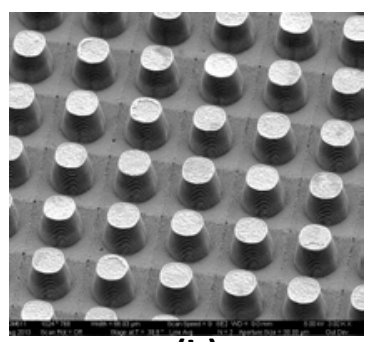

(b)

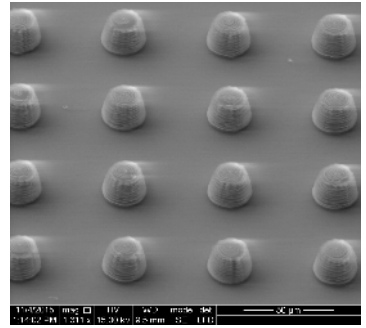

(d)

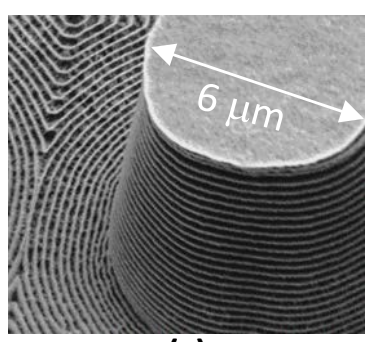

(c)

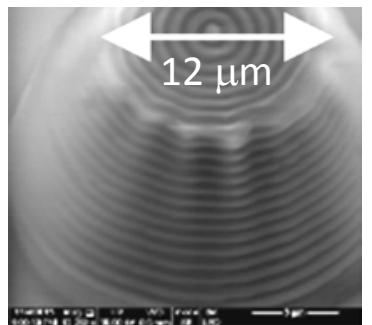

(e)
Figure 4. (a) An optical image of fabricated metal and PDMS non-wetting surfaces. Low and high magnification scanning microscope images of (b), (c) metal ( $\left.w_{\text {Nano }}=50 \mathrm{~nm}, g_{\text {Nano }}=125 \mathrm{~nm}\right)$ and (d), (e) PDMS ( $\left.w_{\text {Nano }}=500 \mathrm{~nm}, g_{\text {Nano }}=500 \mathrm{~nm}\right)$ structures.

\section{CHARACTERIZATION}

Characterization of the fabricated surfaces is performed based on contact angle analysis. Both static contact angle (SCA) and contact angle hysteresis (CAH, difference between advancing and receding angle) are measured using a goniometer (Model 200, Rame-Hart). Lower SCA and CAH represent smaller energy required to change the position of a liquid droplet, and thus, represents decreased solid-to-liquid friction [14]; this required energy can be characterized by measuring the sliding angle. Superhydrophobicity is often characterized by SCA larger than $150^{\circ}, \mathrm{CAH}$ smaller than $10^{\circ}$ and sliding angle smaller than $10^{\circ}$.
Planar

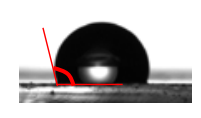

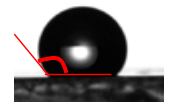

$\operatorname{Micro}(M)$

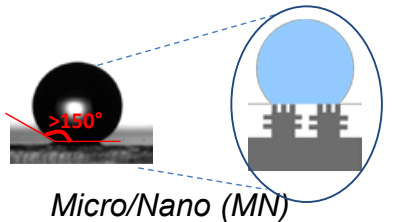

Micro/Nano (MN)
Figure 5. Magnified view of droplets $(5 \mu L)$ on respective PDMS surface structures for static contact angle measurements. The "Planar" sample is cured PDMS without any surface textures. The cartoon describes a droplet supported by the micro/nanostructures, forming a Cassie state.

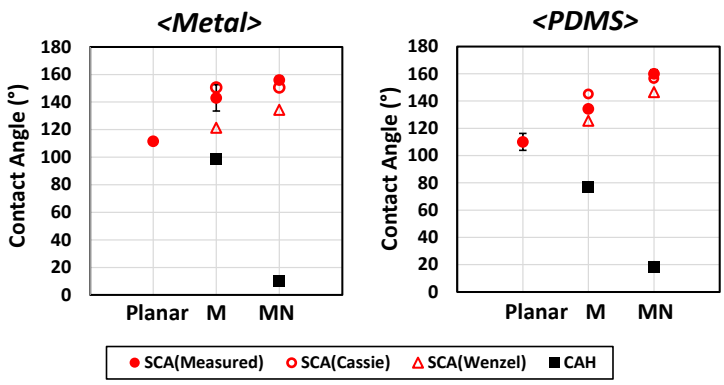

Figure 6. Measured static contact angle (SCA) and hysteresis (CAH) for metal (left) and PDMS (right). Calculated static angles based on Cassie and Wenzel model using equation (1) and (2) are plotted together. Sample dimensions: Metal, M: $w_{\text {Micro }}=20 \mu \mathrm{m}, g_{\text {Micro }}=40 \mu \mathrm{m}, h_{\text {Micro }}=18 \mu \mathrm{m}$, PDMS, $M$ : $w_{\text {Micro }}=15$ $\mu \mathrm{m}, g_{\text {Micro }}=15 \mu \mathrm{m}, h_{\text {Micro }}=8 \mu \mathrm{m}$. For both Metal, MN and PDMS, $M N$ : $w_{\text {Nano }}=500 \mathrm{~nm}, g_{\text {Nano }}=500 \mathrm{~nm}, h_{\text {Nano }}=450 \mathrm{~nm}$.

The static contact angle of a droplet on a textured surface can be modeled using a Cassie $\left(\theta^{\mathrm{c}}\right)$ or Wenzel $\left(\theta^{\mathrm{w}}\right)$ model depending on whether air voids are created beneath a droplet, achieving a stable solid-water-air interface, or not (i.e., formation of a complete solidwater interface). The modeled contact angles are calculated using the equations shown below:

$$
\begin{aligned}
& \text { Cassie model: } \cos \theta^{\mathrm{c}}=f\left(\cos \theta_{0}+1\right)-1 \\
& \text { Wenzel model: } \cos \theta^{\mathrm{w}}=r\left(\cos \theta_{0}\right)
\end{aligned}
$$

where $\theta_{0}$ is Young's contact angle defined on the smooth surface of a material (i.e., measured from a silane-deposited, smooth nickel surface, or a cured PDMS surface (Figure 6)), $r$ is the actual, total surface area divided by the apparent surface area, and $f$ is the solidliquid contact area divided by the surface area underneath the droplet.

Achieving a stable Cassie state is very important to realize superhydrophobic surfaces, since the reduced water-solid contact area leads to the reduced solid-water friction; consequently, there is more chance for a water droplet to roll off from the surface, resulting in useful properties such as self-cleaning. Based on a thermodynamic analysis, it can be shown that the Cassie state is more likely to be energetically favorable compared to the Wenzel state, and therefore, thermodynamically stable, for surface structures with (1) smaller periodicities and (2) higher aspect ratios [13]; hierarchical surfaces bearing such nanostructures on the microstructures are even more desirable since the water-solid contact fraction is drastically decreased due to the presence of the microstructures while high Cassie state stability is still achieved by the nanostructures [1]

This is confirmed by comparing the measurement and calculation results (Figures 5,6). For both metal and PDMS surfaces, the SCA on the single-scale microstructures falls within the contact angles predicted by the Wenzel and Cassie models. This means a stable Cassie state is not achieved with the single-scale structures even at a low liquid pressure $(\sim 100 \mathrm{~Pa})$ induced by a droplet with a small volume $(5 \mu \mathrm{L})$. On the other hand, the high SCA of $160^{\circ}$ measured from the hierarchical structures corresponds to the Cassie model. The measured CAH from the hierarchical surfaces are much lower than the measurements from the single-scale structures $(>$ $80^{\circ}$ ). The high SCA and low CAH of hierarchical surfaces correspond to smaller measured sliding angles (i.e., the angles at which a droplet of a given volume, $10 \mu \mathrm{L}$, begins to roll off from the substrate: $9^{\circ}-12^{\circ}$ ) compared to the measurements from the single- 
scale structures $\left(>90^{\circ}\right.$, droplets not rolling off even from verticallyoriented surfaces). In summary, both metal and PDMS hierarchical surfaces exhibit superhydrophobicity due to their multiscale architectures.

The utility of the fabricated superhydrophobic surfaces is demonstrated by a droplet impact test and a self-cleaning experiment. Droplets are dispensed from a pipette toward a horizontal, hierarchical surface from a distance $(\sim 1.5 \mathrm{~cm})$. Figure 7 captures a single droplet after impact bouncing from the PDMS surface while its spherical shape is preserved. Due to the strong nonwetting nature of the patterned region, the bounced droplet moves toward the edge of the sample where no surface textures are present. Self-cleaning of the hierarchical surfaces is demonstrated by using iron powder (100-200 mesh) "contaminants." After the powders are sprayed on the angled surfaces (Figure 8(a),(b), inserts), water droplets are gently placed on the surfaces. The droplets quickly roll off from the surfaces, removing the contaminants and leaving the clean traces behind (Figure 8(a),(b)). A drastic particle density contrast between the "cleaned" and "uncleaned" areas is observed in the magnified images (Figure 8(c),(d)).
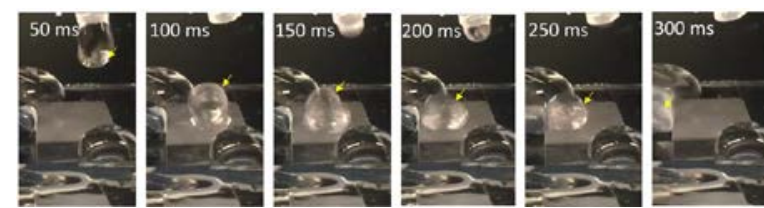

Figure 7. A droplet bouncing on a hierarchical PDMS surface. Note that the droplet shape remains spherical after impact (at $250 \mathrm{~ms}$ ).

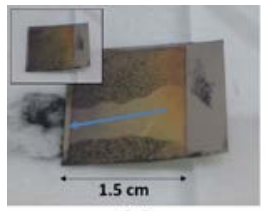

(a)

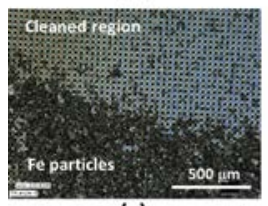

(c)

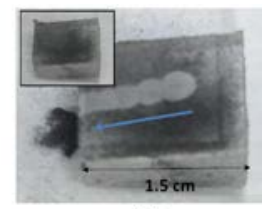

(b)

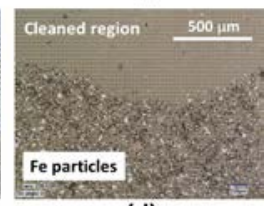

(d)
Figure 8. (a) Metallic and (b) PDMS surfaces after water droplets are dispensed and rolled off from the surfaces (Arrows). Figures (c), (d) are magnified images near the edges of the "cleaned" areas.

\section{CONCLUSION}

Multilayer electrodeposition guided by micromachined templates was presented as a scalable route to superhydrophobic surface structures. The surface chemistry of the etched multilayers was modified (i.e., silanization) to create metallic superhydrophobic surfaces. Such surfaces were utilized as master molds to produce PDMS superhydrophobic surfaces via replica molding. Both surfaces showed characteristics representative of superhydrophobic surfaces: high static contact angle reaching $160^{\circ}$, small contact angle hysteresis less then $10^{\circ}$, and low sliding angle. Since the geometry of the microstructures, density or aspect ratios of nanostructures, and surface chemistry are widely controllable, this process could be extended to multiple applications, such as spatially anisotropic wettabilities for directional fluid control, or extreme hydrophilicities for fluid wicking.

\section{ACKNOWLEDGEMENT}

Microfabrication was carried out in part in the Singh Center for Nanotechnology at the University of Pennsylvania. The authors thank Mr. Gaoxiang Wu and Dr. Shu Yang at the University of Pennsylvania for assistance with contact angle measurements.

\section{REFERENCES}

[1] Y. Su, et al., "Nano to Micro Structural Hierarchy is Crucial for Stable Superhydrophobic and Water-Repellent Surfaces," Langmuir, 26, 7, (2010).

[2] X.-Q. Feng, X. Gao, Z. Wu, L. Jiang, and Q.-S. Zheng, "Superior Water Repellency of Water Strider Legs with Hierarchical Structures: Experiments and Analysis," Langmuir, 23, 9, (2007).

[3] T. Liu, and C.-J. Kim, "Doubly Re-Entrant Cavities to Sustain Boiling Nucleation in FC-72," Technical Digest of the IEEE $28^{\text {th }}$ IEEE International Conference on Micro Electro Mechancial Systems, Estoril, Portugal, 1/18-1/22, (2015), pp. 1122-1124.

[4] M. Raza, H. Zandvliet, B. Poelsema, and E. Kooij, "Hydrophobic Ssurfaces with Tunable Dynamic Wetting Properties via Colloidal Assembly of Silica Microspheres and Gold Nanoparticles," Journal of Sol-Gel Science and Technology, 74, 2, (2015).

[5] Y. Xiu, L. Zhu, D. W. Hess, and C. Wong, "Hierarchical Silicon Etched Structures for Controlled Hydrophobicity/Superhydrophobicity," Nano Letters, 7, 11, (2007).

[6] F. Zhang, and H. Y. Low, "Anisotropic Wettability on Imprinted Hierarchical Structures," Langmuir, 23, 14, (2007).

[7] S.-J. Choi, K. Y. Suh, and H. H. Lee, "Direct UV-Replica Molding of Biomimetic Hierarchical Structure for Selective Wetting," Journal of the American Chemical Society, 130, 20, (2008).

[8] T.-H. Kim, et al., "Simple and Cost-Effective Fabrication of Highly Flexible, Transparent Superhydrophobic Films with Hierarchical Surface Design," ACS Applied Materials \& Interfaces, 7, 9, (2015).

[9] X.-S. Zhang et al., "Single-Step Fabrication of Superhydrophobic Micro/Nano Dual-Scale PDMS Film Replicated from Ultra-Low-Surface-Energy Mold," Technical Digest of the IEEE $26^{\text {th }}$ IEEE International Conference on Micro Electro Mechancial Systems, Taipei, Taiwan, 1/20-1/24, (2013), pp. 331-334.

[10] R. Hensel et al., "In Situ Experiments to Reveal the Role of Surface Feature Sidewalls in the Cassie-Wenzel Transition," Langmuir, 30, 50, (2014).

[11] M. Kim, J. Kim, and M. Allen, "Nanopatterned Surfaces Based on Template-Assisted Multilayer Electrodeposition," Small, $11,16,(2015)$.

[12] T. W. Odom, J. C. Love, D. B. Wolfe, K. E. Paul, and G. M. Whitesides, "Improved Pattern Transfer in Soft Lithography Using Composite Stamps," Langmuir, 18, 13, (2002).

[13] W. Li, and A. Amirfazli, "A Thermodynamic Approach for Determining the Contact Angle Hysteresis for Superhydrophobic Surfaces," Journal of Colloid and Interface Science, 292, 1, (2005).

[14] C. Furmidge, "Studies at Phase Interfaces. I. The Sliding of Liquid Drops on Solid Surfaces and A Theory for Spray Retention," Journal of Colloid Science, 17, 4, (1962).

\section{CONTACT}

*M. Kim, tel:+1-404-630-6262; novamagic@gmail.com

M. Allen, tel:+1-215-898-5901; mallen@upenn.edu 